\title{
Ein Verfahren zur Ermittlung der Bilanz einverleibter Stoffe ohne Kenntnis der Ausscheidung
}

\author{
Von \\ F. H. Dost und E. Gladtke \\ Aus der Kinderklinik der Justus Liebig-Universität Gießen (Direktor: Prof. Dr. F. H. Dost)
}

(Der Schriftleitung zugegangen am 8. Dezember 1962)

\begin{abstract}
Es wird ein Verfahren beschrieben, das die Ermittlung der Resorptionsbilanz eines applizierten Medikaments in einfacher Weise gestattet. Es basiert auf der mehrfachen analytischen Bestimmung der Konzentration des applizierten Stoffes im Blute. Trägt man die gefundenen Konzentrationen in Abhängigkeit von der Zeit in ein Diagramm ein, so resultieten Konzentrationsverlaufskurven. Unabhängig von Applikationsform und -dauer sind die Flächen unter diesen Konzentrationsverlaufskurven bei gleicher Dosis und gleicher Person gleich. Bei Zufuhr verschiedener Dosen verhalten sich unabhängig von der Applikationsart und damit unabhängig von der Form der Kurve die Flächen unter den Kurven wie die Dosen, sofern vollständige Absorption erfolgt. Damit entspricht das Verhältnis der Größe der Flächen der Absorptionsrate. Bilanzuntersuchungen sind damit bei analytisch nachweisbaren und injizierbaren Stoffen nicht mehr erforderlich. Vergleichende Blutspiegeluntersuchungen haben die gleiche Aussagekraft.

A simple method is described for the determination of the absorption balance of medicaments. It is based on several determinations of the substance in the blood. Concentration plotted against time then gives a curve for the time course of the concentration. Irrespective of mode and duration of administration, the areas under these time/concentration curves are the same for each individual and the same dose. The areas under the curves vary directly with the dose and are independent of the mode of administration (and thus the shape of the curve), providing complete absorption occurs. The rate of increase in curve area is thus proportional to the rate of absorption. Belance experiments are therefore not necessary with injected, measurable substances. Determinations of blood levels gives the same information.
\end{abstract}

Es wird eine Methode dargestellt, die es gestattet, an Hand von Konzentrationsbestimmungen im Blut den stofflichen Umsatz von Medikamenten innerhalb des Organismus zu ermitteln, obne deren Verteilung auf die verschiedenen Organe und Gewebe sowie deren Ausscheidung in Exkreten oder Sekreten feststellen oder messen zu müssen.

\section{Pharmakokinetische Grundlagen}

Es interessieren uns hier ausschließlich die nach Belastung des Organismus mit einem körperfremden Stoff resultierenden Konzentrationsabläufe im Blut. Trägt man im Diagramm mit einfach-arithmetisch geteiltem Raster die Konzentrationen in Abhängigkeit von der Zeit ein, so resultieren Konzentrationsverlaufskurven. Die Flächeninhalte $(F)$ unter diesen Kurven stellen hierbei einen Maßstab für die in dem betrachteten Zeitabschnitt auf dem Weg über das Blut umgesetzten Stoffmengen dar. Sie sind mit derumgesetztenDosis $(D)$ durch ein Verhältnis einfacher Proportionalität, entsprechend:

$$
D=\text { const } \cdot F
$$

verbundẹn (Dost $(1,2)$ ), bżw. sogar durch ein Verhältnis gegenseitiger af finer Abbildbarkeit (Dost (3)). Diese Beziehung gilt unabhängig davon, ob die Konzentrationskurve einer mathematischen Analyse zugängig ist (1) oder nicht (2), sofern nur hinsichtlich des applizierten Stoffes ein Diffusionsgleichgewicht bzw. ein "Abflußgleichgewicht" (4) zwischen. Kreislaufflüssigkeit und Geweben angenommen werden kann.

a) Matbematische Flächenbestimmung: Nicht selten erhält man, z. B. nach intravaskulärer Verabreichung eines Stoffes Konzentrationsabläufe im Blut, die als mathematische Funktionen formuliert werden können (Abbildung 1). Auf welche Weise in diesen Fällen auch

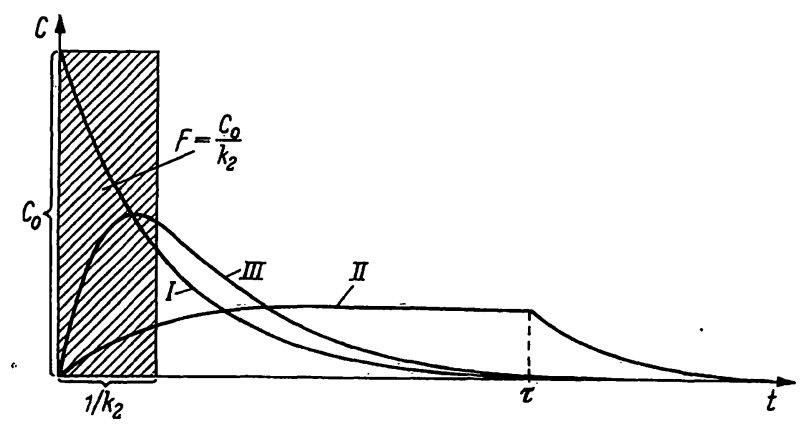

Abb. 1

Konzentrationsverläufe: I: bei rascher intravenöser Gabe II: bei intravenöser Dauerinfusion, beendigt bei $t=\tau$

III: nach nicht-intravaskulärer Verabreichung und vollständiger Absorption.

(Bei gleicher Dosis lassen sich die Flächen unter den Kurven I-III durch das abgebildete Rechteck $\frac{c_{0}}{k_{2}}$ darstellen.) 
immer die Applikation des Stoffes erfolgt sein mag, seine vollständige Aufnahme in das Transportorgan des Blutes ergibt sich stets dadurch zu erkennen, daß die Flächen unterhalb der jeweiligen Kurve durch die stets gleiche Fläche $F$ des Rechteckes:

$$
F=\frac{c_{0}}{k_{2}}
$$

dargestellt werden können.

$c_{0}$ : (fiktive) Anfangskonzentration des betrachteten Stoffes im Blut, welche zu beobachten sein würde, wenn sich zur Zeit $t=0$ bereits ein Diffusionsgleichgewicht im Verteilungsraum des applizierten Stoffes, bezogen auf die gesamte transportierte Dosis, eingestellt haben würde; $k_{2}$ : Eliminationskonstante („ClearanceKoeffizient", "Slope“, „relative Umsatzrate", „Turnoverrate" usw.) des betreffenden Stoffes. Die Dimension der Fläche $F$ im absoluten Maßsystem ist: $l^{-3} m \cdot t$.

b) Pragmatische Flächenbestimmung: Ist der erhaltene Konzentrationsablauf einer mathematischen Interpretation nicht zugänglich oder zieht man es vor, auf das mathemathische Kalkül zu verzichten, so kann man die unter der Konzentrationskurve befindliche Fläche auch mechanisch ausmessen, etwa unter Verwendung eines Polarplanimeters, oder indem man die Flächen aus dem Diagramm herausschneidet und das Gewicht des anfallenden Papiers auf einer Analysenwaage feststellt. Man erhält auf diese Weise Flächenwerte $F_{P}$ bzw. $F_{G}$ der Dimension $l^{2}$ bzw. $g$, was beim Umrechnen von $F_{P}$ und $F_{G}$ in $F \mathrm{zu}$ berücksichtigen ist.

Auf welche Weise man $F$ nun auch ermittelt haben mag, man kann die erhaltenen Werte nun ohne weiteres in die aus $G l$. (1) abgeleitete dimensionslose Beziehung:

$$
\frac{D_{1}}{D_{2}}=\frac{F_{1}}{F_{2}}
$$

einsetzen, wo $D_{1}$ und $D_{2}$ zwei verschiedene Dosen des gleichen Stoffes und $F_{1}$ sowie $F_{2}$ die beiden, zu jeder Dosis gehörenden Flächen darstellen, sofern man nur auf Dimensionsgleichheit bedacht ist. Die mit Gl. (3) dargestellte Funktion gilt selbstverständlich nur für den Fall, daß die Dosis ohne jeden Verlust tatsächlich auch im Blut erscheint. Übertragen auf das pharmakokinetische Geschehen können wir daher Gl. (3) ohne weiteres als gültig für die Verhältnisse bei intravaskulärer, d. h. (im allgemeinen) bei intravenöser Applikation der Dosis betrachten. Bieten wir hingegen den betrachteten Stoff dem Organismus auf dem Weg über die Resorption (subkutane, intramuskuläre Injektion, per os, durch Sonde, rektal usw.) an, so muß die im Blut erscheinende Dosis nicht ohne weiteres der verabreichten Menge gleich sein, wenn infolge von chemischen Umsetzungen am Ort des Depots bzw. durch teilweise Zerstörung des Stoffes vor erfolgter Resorption bzw. auch einfach infolge unvollständiger Resorption ein Teil der verabreichten Menge nicht in das Blut aufgenommen wird und daher auch nicht in die allgemeine Bilanz des Umsatzes eingehen kann. Wir wollen diesen Teil der Dosis jetzt mit $D_{n a}$ (nicht absorbiert $\left.{ }^{1}\right)$ ), den im Blut erscheinenden Teil mit $D_{a}$ (absorbiert) und die verabreichte Ausgangs- oder Total-Dosis mit $D_{l o t}$ bezeichnen, entsprechend:

$$
D_{\text {lot }}=D_{a}+D_{n a}
$$

Da nun $D_{\text {lot }}$ unter allen Umständen stets dann im Blut erscheint, wenn wir die Gesamtmenge des Stoffes intravaskulär (z. B. intravenös) injizieren, gilt für diesen Fall: $D_{t v}=D_{l o t}=D_{a}$. Schreiben wir nunmehr statt Gl. (3):

$$
\frac{D_{a}}{D_{i v}}=\frac{F_{a}}{F_{i v}}
$$

so kommen wir zu einer einfachen Proportionalität zwischen den Flächen einerseits und den Dosen andererseits, welche nach intravaskulärer (intravenöser) bzw. nicht-intravaskulärer Applikation der gleichen Ausgangsdosis des gleichen Stoffes beim gleichen Individuum im Blut erscheinen.

Will man also ermitteln, welcher Prozentsatz $p$ der Ausgangsdosis eines Stoffes nach beliebiger, nicht intravaskulärer Applikation resorbiert wurde und in unverändertem Zustand über das Blut in Umlauf gesetzt wurde, so gilt:

$$
P_{\left(D_{a} / D_{i v}\right)}=\frac{F_{a}}{F_{i v}} \cdot 100,
$$

entsprechend erhält man für $D_{a}$ in Gewichtseinheiten:

$$
D_{a}=\frac{F_{a}}{F_{i v}} \cdot D_{i v} \text {. }
$$

\section{Experimentelle Prüfung}

a) Vergleich der Flächen bei Variation der Geschwindigkeit der intravenösen $Z$ ufubr

Zunächst wurden Versuche bei unterschiedlicher Dosierung und rascher intravenöser Injektion vorgenommen (6-Sulfanilamido-2,4-dimethylpyrimidin („Aristamid“, „Elkosin“)). Ein Kind erhielt einmal 450 und das zweite Mal $300 \mathrm{mg}$, also beim zweitenmal $2 / 3=$ $67 \%$ der ersten Dosis, beide Male intravenös. Aus den Kurven wurden $64 \%$ errechnet und $65 \%$ planimetriert (Abbildung 2). Einem anderen Kind verabfolgten wir in gleicher Weise i. v. 360,540 und $720 \mathrm{mg}$, also 100,150 und $200 \%$. Die Flächenwerte ergaben 151 und 213 (planimetriert) bzw. 152 und 220\% (errechnet) (Tabelle 1). Die Flächen verhalten sich in guter Annäherung proportional zur verabfolgten Dosis, es besteht also eine feste Beziehung.

Alsdann haben wir zusammen mit Moros (5) vergleichende Untersuchungen bei unterscbiedlicber intravenöser Injektionsgeschwindigkeit und unveränderter Dosis durchgeführt ( $p$-aminohippursaures $\mathrm{Na}$ („Nephrotest $\left.{ }^{\circ}\right)$ ). Wir applizierten die gleiche Menge dieses Stoffes der gleichen Versuchsperson an verschiedenen Tagen mit verschiedener Geschwindigkeit (rasche

1) Statt „Resorption“ wird im internationalen Schrifttum heute vorwiegend das Synonym „Absorption" benutzt. 


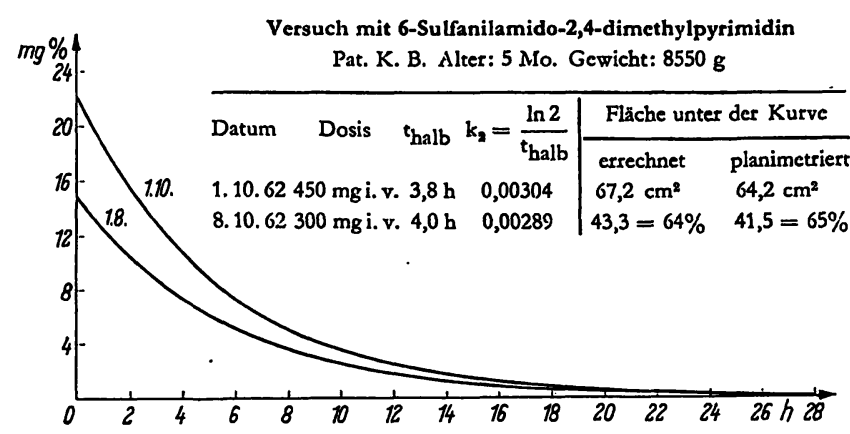

Abb. 2

Konzentrationsverläufe und Flächenauswertung bei intravenöser Gabe verschiedener Dosen

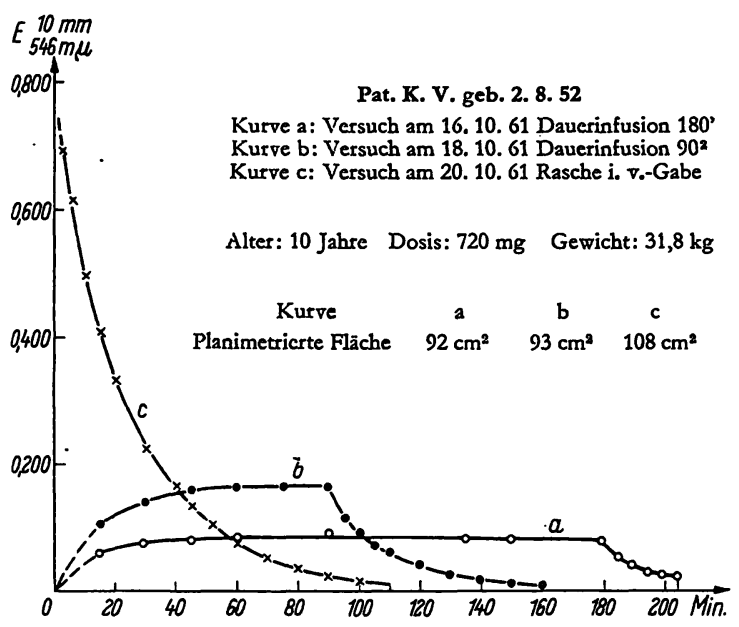

Abb. 3

Konzentrationsverläufe und Flächenauswertung bei verschieden rascher intravenöser Verabfolgung von $\mathrm{PAH}$
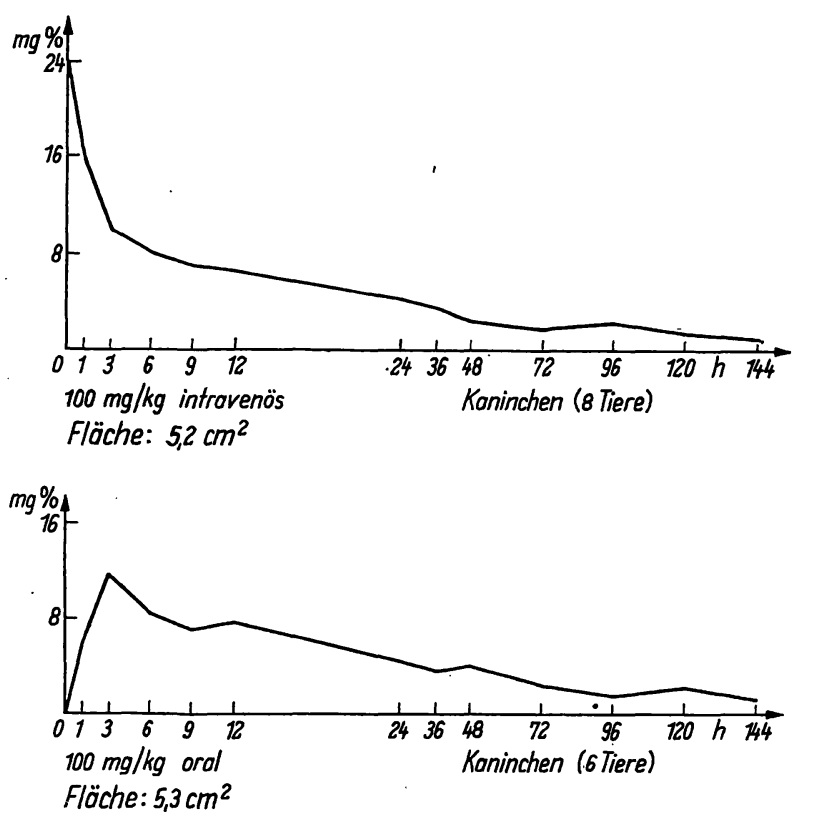

Abb. 4

Vergleich zweier Konzentrationsverläufe und Flächenauswertung nach intravenöser und enteraler Gabe gleicher Mengen von Sulphaphenazol (nach Schmid, Tripod und Gross)
Tab. 1

Daten bei intravenöser Gabe verschiedener Dosen

Kind S. S. geb. 28.11.61 Gew. $7300 \mathrm{~g}$

\begin{tabular}{|c|c|c|}
\hline Datum & Dosis $i$ & Flächeninhalt \\
\hline Datum & Dosis 1. v. & gemessen berechnet \\
\hline
\end{tabular}

11. 9. $62540 \mathrm{mg}=150 \% \quad 540 \mathrm{~cm}^{2}=151 \% \quad 532 \mathrm{~cm}^{2}=152 \%$

15. $9.62720 \mathrm{mg}=200 \% \quad 767 \mathrm{~cm}^{2}=213 \% \quad .767 \mathrm{~cm}^{2}=220 \%$

19. $9.62360 \mathrm{mg}=100 \% \quad 359 \mathrm{~cm}^{2}=100 \% \quad 349 \mathrm{~cm}^{2}=100 \%$

i. v.-Gabe und i. v.-Dauerinfusion mit verschiedenen Geschwindigkeiten). Die Flächen unter den jeweils erhaltenen Kurven ergaben eine gute Übereinstimmung der Werte bei graphischer Bestimmung mit dem Planimeter (Abbildung 3).

\section{b) Prüfung der Vollständigkeit des Absorptions-Vorganges bei nicht-intravenöser $Z$ ufubr}

Es sei hier zunächst auf ein Untersuchungșergebnis hingewiesen, das uns seinerzeit veranlaßte, die eigenen, hier mitgeteilten Arbeiten zu unternehmen. - Es handelt sich dabei um eine Mitteilung von SCHMID, TrIPOD und Gross (6), die in Versuchen am Kaninchen beim Vergleich von kolorimetrischen und radiometrischen Messungen an Hand sehr genauer Bilanzuntersuchungen die vollständige Absorption des Sulfonamids "Orisul" (Sulphaphenazol) nachgewiesen haben. Beim Planimetrieren der unter den abgebildeten Blutspiegelkurven befindlichen Flächen fiel damals dem einen von uns (D.) auf, daß dieselben bei intravenöser und enteraler Verabreichung mit 5,2 bzw. $5,3 \mathrm{~cm}^{2}$ ) untereinander gleich waren. Die Bedeutung dieses Ergebnisses blieb den Autoren verborgen. Auf die Wiedergabe der entsprechenden Diagramme möchten wir an dieser Stelle nicht verzichten. Aus diesen (Abbildung 4) geht hervor, daß Flächengleicbheit zwischen intravenöser und nichtintravenöser Applikation besteht, wenn jeweils die gleiche Dosis verabfolgt wurde, und in beiden Fällen vollständig im Blut erscheint, wie dies im übrigen die gleichzeitig ausgeführten mühevollen Bilanzuntersuchungen der genannten Autoren ergeben hatten.

Damit wäre zunächst gezeigt, daß gleiche Dosis bei hundertprozentiger Absorption die gleiche Fläche ergibt. Kehren wir diesen Satz um, so sagt er aus, daß eine Differenz der Flächengröße zwischen enteraler und intravenöser Applikation anzeigt, daß die Absorption aus dem Darm nicht vollständig ist. Eine Flächendifferenz ist demnach Ausdruck einer unvollständigen Absorption.

Unsere weiteren Untersuchungen haben wir mit 6-Sulfanilamido-2,4-dimethylpyrimidin („Aristamid“, „Elkosin") unternommen, da es eine brauchbare. Eliminations-Halbwertszeit zu haben schien. Die Bestimmung erfolgte im Serum nach dem Prinzip von Bratton-Marshall (7) durch Diazotierung nach Enteiweißung photometrisch mit einer Halbmikro-Methode mit Hilfe der Sanzschen Apparatur und selbstge-

1) Sämtliche Flächenmaße sind einheitlich in $\mathrm{cm}^{2}$ angegeben (planimetrische oder mathematische Auswertung der Originaldiagramme). Die Verkleinerung der Flächen für die Wiedergabe beim Druck konnte selbstrerständlich vorher keine Berücksichtigung durch uns finden. 
fertigter Konstriktionspipetten aus Polyäthylen nach Matrenheimer (8). Wir benötigen für eine Doppelbestimmung $0,025 \mathrm{ml}$ Serum.

Es wurden zunächst zur Ermittlung der Fläche bei parenteraler Gabe eine geeignete Menge (etwa $50 \mathrm{mg} / \mathrm{kg}$ ) intravenös verabfolgt und dann etwa 4 bis 6 Bestimmungen in ein- bis zweistündigen Abständen durchgeführt. Da man beim Auftragen einer Exponentialfunktion im halblogarithmischen Netz eine Gerade bekommt, genügen theoretisch $\cdot z$ wei Werte, die bei intravenöser Gabe im Verteilungsvolumen ermittelt werden, um die Vergleichskurve zu erhalten. Wir empfehlen trotzdem 4 bis 5 Messungen, da gewisse Fehlermöglichkeiten dann besser übersehbar sind. Die Kurve wird auf Millimeterpapier mit arithmetischer Teilung beider Ordinaten transformiert, die Fläche unter der Kurve mit Hilfe eines geeigneten Planimeters ermittelt oder errechnet und mit der (stets planimetrisch ermittelten) Fläche unter der Kurve verglichen, welche man nach nicht-intravaskulärer Verabreichung erhalten hat. Einige Tage später haben wir den Stoff in der vorgesehenen Menge per Sonde in den Magen gegeben bzw. intravenös verabfolgt. Durch häufigere Analysen wurde versucht, eine repräsentative Kurve zu erhalten.

Die Auswertung sei an folgendem Beispiel erläutert: (Abbildung 5). Die Kurve A gibt die Elimination nach intravenöser Gabe von $510 \mathrm{mg}$ Sulfonamid bei einem gesunden Kind wieder. Kurve B ist nach Gabe derselben Menge per Sonde ein paar Tage später gewonnen worden. Es konnte nur der erste Teil der Kurve aufgezeichnet werden, und zwar über eine Zeit von 270 Minuten. Die Kurven wurden mit dem Planimeter ausgemessen. Der sozusagen abgeschnittene Rest der Kurve läßt sich mit Leichtigkeit nach Gl. (2) ermitteln. Die Eliminationskonstante $k_{2}$ ist nach der Formel

$$
k_{2}=\frac{\ln 2}{t_{\text {halb }}}=\frac{0,693}{t_{\text {halb }}}
$$

zu berechnen, $t_{\text {halb }}$ wurde graphisch mit Hilfe der auf halblogarithmischem Raster aufgetragenen Eliminationskurve ermittelt. Zur Kontrolle haben wir auch die Anfangsteile der Kurve A berechnet und mit dem Ergebnis der Planimetrie verglichen. Die Abweichung beträgt hier weniger als $2 \%$. Die Summe der beiden Kurventeile ist als $F$ von $t_{0}$ bis $t_{\infty}$ mit 765,6 bzw. $740,6 \mathrm{~cm}^{2}$ angegeben. Die Differenz beträgt $3 \%$. In der Tabelle 2 sind die Mittelwerte eines gleich-

Tab. 2

Dạten bei gleicher Dosis und unterschiedlicher Zufuhr Mittelwerte von einem Versuch mit 3 Säuglingen mit 6-Sulfanilamido-2,4-dimethylpyrimidin

Alter: 7 Monate Gewicht: $4920 \mathrm{~g}$ Dosis: $480 \mathrm{mg}$

\begin{tabular}{rcccc}
\hline Datum & Applikation & $c_{0}$ & $t_{\text {halb }}$ & Fläche \\
\hline 5.9 .62 & intravenös & $31,5 \mathrm{mg} \%$ & $3,5 \mathrm{~h}$ & $111 \mathrm{~cm}^{2}$ \\
16.9 .62 & per Sonde & $32,5 \mathrm{mg} \%$ & $3,5 \mathrm{~h}$ & $114 \mathrm{~cm}^{2}$
\end{tabular}

Versuch mit 6-Sulfanilamido-2,4-dimethylpyrimidin Pat. J. H. Alter: 8 Monate Gewicht: $8740 \mathrm{~g}$ Dosis: $510 \mathrm{mg}$ Datum Applikation F von $t_{0}-t_{270}$ F v.t $t_{270}{ }^{-t} \infty \quad \cdot F v . t_{0}-t_{\infty}$ gemessen berechnet berechnet ber. + gem. berechnet

\begin{tabular}{lllllll}
\hline Kurve A 20.8 .62 i. v. & $429,6 \mathrm{~cm}^{2} \quad 422 \mathrm{~cm}^{2} \quad 336 \mathrm{~cm}^{2} \quad 765,6 \mathrm{~cm}^{2} \quad 758 \mathrm{~cm}^{2}$
\end{tabular} Kurve B 24. 8. $62 \mathrm{per}$ Sondc $420,6 \mathrm{~cm}^{2}$ - $320 \mathrm{~cm}^{2} 740,6 \mathrm{~cm}^{2}$ -

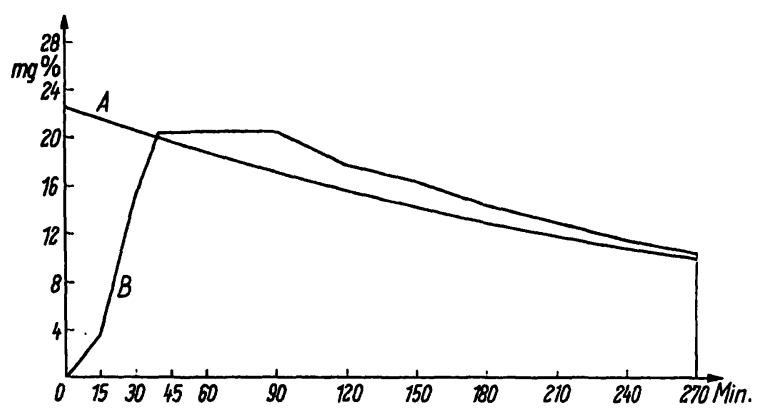

Abb. 5

Konzentrationsverläufe und Flächenauswertung bei intravenöser und enteraler Gabe der gleichen Dosis

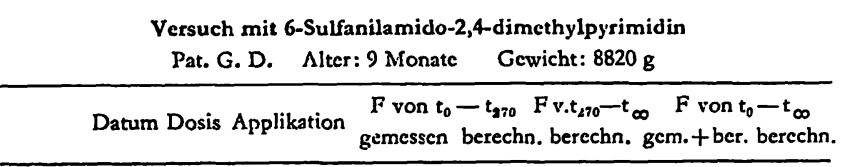

Kurve A $2.9 .62450 \mathrm{mg}$ intravenös $481,0 \mathrm{~cm}^{2} \quad 461 \mathrm{~cm}^{2} \quad 502 \mathrm{~cm}^{2} \quad 983,0 \mathrm{~cm}^{2} \quad 963 \mathrm{~cm}^{2}$ Kurve B $7.9 .62300 \mathrm{mg}$ per Sonde $329,4 \mathrm{~cm}^{2}-374 \mathrm{~cm}^{2} 703,4 \mathrm{~cm}^{2}-$

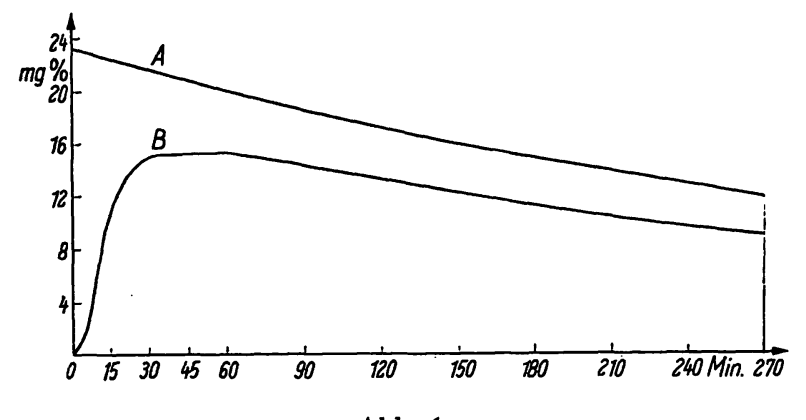

Abb. 6

Konzentrationsverläufe und Flächenauswertung bei Gabe verschiedener Dosen intravenös und per Sonde

artigen Versuches an drei gleichalten und gleichschweren gesunden Säuglingen aufgetragen. Die Flächen stimmen auch hier überein. Wir können somit feststellen, daß bei intravenöser und bei oraler Gabe gleicher Dosen die Flächen unter den Kurven gleich sind. Der von uns applizierte Stoff ist also vollständig absorbiert worden. Im letzten Versuch haben wir $450 \mathrm{mg}$ intravenös und eine kleinere Dosis von $300 \mathrm{mg}=67 \%$ per Sonde verabfolgt (Abbildung 6). Die Fläche unter der Kurve nach enteraler Applikation wurde mit $71 \%$ der anderen Fläche ermittelt. Auch hier verhalten sich die Flächen proportional zur verabfolgten Dosis; der Fehler beträgt $+5 \%$.

Es ist damit bewiesen, daß die Größe der Fläche dosisabhängig ist und $\mathrm{da} B$ an Hand vergleichender Blutspiegelkurven eine Aussage über die Absorptionsrate möglich ist. Die absorbierte Dosis $D_{a}$ verhält sich zur intravenös verabfolgten Dosis $D_{i v}$ wie die Größen der Flächen unter den jeweiligen Kurven: $\frac{D_{a}}{D_{i v}}=\frac{F_{a}}{F_{i v}}$. 
Zusammenfassend kann gesagt werden, daß zur Bestimmung der Absorptionsrate eines Stoffes lediglich der Vergleich der Flächen unter den Kurven nach intravenöser und nach nicht-intravenöser Gabe derselben Dosis bei derselben Versuchsperson nötig ist.
Die Relation der Flächen entspricht der Absorptionsrate. Damit ist das Verfahren zur pharmakokinetischen Prüfung der Vollständigkeit der Absorption geeignet und experimentell bestätigt.

\title{
Literatur
}

1. Dosr, F. H., Klin. Wschr. II, 655 (1958). - 2. Dost, F. H., Antibiotica et chemotherapia, im Druck. - 3. Dosr, F. H., Dtsch. med. Wschr. II, 1833 (1962). - 4. Moeller, J. und L. Aвt, Klin. Wschr. I, 340 (1952). - 5. GladtKe, E. und E. Mohos, Veröffentlichung in Vorbereitung. - 6. SCHMIDT, K., S. TRIPOD und F. Gross, Klin. Wschr. 38, 862 (1960). - 7. Bratron, A. C. und E. K. Marshatr, J. Biol. Chem., Baltimore, 128, 537 (1939). 8. Matrenhermer, H., Mikromethoden für das klinisch-chemische und biochemische Laboratorium, Walter de Gruyter \& Co., Berlin (1961), S. 9-12.
Professor Dr. F. H. Dost

Direktor der Universitätskinderklinik 63 Gießen, Klinikstr. 28

\section{Untersuchungen über das Verhalten der Plasmalipoide bei idiopathischer Hypercholesterinämie, unter besonderer Berücksichtigung der Cholesterinester ${ }^{1)}$}

\author{
Von \\ NePOMUK ZÖLLNER \\ Aus der Medizinischen Poliklinik der Universität München (Diriktor: Prof Dr. W. Seitz). \\ (Der Schriftleitung zugegangen am 1. September 1962)
}

\begin{abstract}
Es wird ein System von Lipoidanalysen beschrieben, das es gestattet, aus einem Lipoidextrakt von $1 \mathrm{ml}$ Serum alle wesentlichen Lipoidgruppen mit Ausnahme der freien Fettsäuren im Doppel zu bestimmen. Die Analyse der Cholesterinester bei Fällen von idiopathischer Hypercholesterinämie ergibt eine normale Verteilung der Cholesterinesterfettsäuren. Dies wird als Beweis dafür angesprochen, daß eine Störung des Cholesterinesterstoffwechsels bei der idiopathischen Hypercholesterinämie nicht vorliegt. Eine vergteichende Untersuchung über die quantitative Bestimmung der Cholesterinester mittels Dünnschicht- oder Gaschromatographie ergibt gute Übereinstimmung beider Methoden.
\end{abstract}

A method is described by which all important groups of lipids except free fatty acids can be determined in duplicate in the lipid extract from $1 \mathrm{ml}$ serum. The analysis of cholesterol esters in idiopathic hypercholesterolaemia gives a normal distribution of cholesterol fatty acids. This is evidence that cholesterol ester metabolism is not disturbed in idiopathic hypercholesterolaemia. Comparison of quantitative cholesterol ester determination by thin layer and gas chromatography shows good agreement.

Die idiopathische familiäre Hypercholesterinämie ist eine angeborene Stoffwechselstörung auf der Grundlage eines genetischen Defektes. Die Grundzüge der Klinik dieser nicht allzu seltenen Krankheit sind durch die klassischen Beschreibungen von Thannhauser und Magendantz (1) sowie Thannhauser (2) dargestellt. Auch die Genetik ist weitgehend bekannt (FrEDRICKSON (3)), wenngleich die erhobenen Befunde noch nicht einheitlich gedeutet werden. Die Abgrenzung der Krankheit gegen die idiopathische Hyperlipämie macht nur gelegentlich noch Schwierigkeiten.

1) Dem Andenken meines verehrten Lehrers Sregrimind. ThanNHAUSER, gestorben am 18. Dez. 1962, gewidmet.
Über die Natur des der idiopathischen familiären Hypercholesterinämie zugrundeliegenden Stoffwechseldefektes ist man noch völlig im Dunkeln. Dies ist verständlich, weil bislang nur bekannt ist, $\mathrm{da}$ B Cholesterin und die Summe seiner Ester in normalen Proportionen vermehrt sind (2). Daneben beobachtet man eine Vermehrung der Phosphatide $(3,4,5)$. und - häufig - der Carotinoide (6). Alle Angaben überLipoidvermehrungen bei der Krankheit beziehen sich also auf Substanzgruppen. Hinweise auf die Lage eines Stoffwechselblocks sind aber erfahrungsgemä $B$ in erster Linie aus der Anhäufung einer individuellen Substanz zu erwarten. Daraus ergibt sich die Forderung, die Analytik bis zur Bestimmung einzelner Lipoide voranzutreiben. Ent- 